\title{
Integrable vortex dynamics in anisotropic planar spin liquid model
}

\author{
Zeynep Nilhan Gurkan, Oktay Pashaev* \\ Department of Mathematics, Izmir Institute of Technology, Gulbahce koyu, Urla, Izmir 35430, Turkey
}

Accepted 10 November 2006

\begin{abstract}
The problem of magnetic vortex dynamics in an anisotropic spin liquid model is considered. For incompressible flow the model admits reduction to saturating Bogomolny inequality analytic projections of spin variables, subject the linear holomorphic Schrödinger equation. It allows us to construct $N$ vortex configurations in terms of the complex Hermite polynomials. Using complex Galilean boost transformations, the interaction of the vortices and the vortex chain lattices (vortex crystals) is studied. By the complexified Cole-Hopf transformation, integrable $N$ vortex dynamics is described by the holomorphic Burgers equation. Mapping of the point vortex problem to $N$-particle problem, the complexified Calogero-Moser system, showing its integrability and the Hamiltonian structure, is given.
\end{abstract}

(C) 2006 Elsevier Ltd. All rights reserved.

\section{Introduction}

As is well known the main ideas of Descartes vortex theory are represented in "Discours de la methode" (1637) and in a capital work "Principia Philosophiae" (1644). The Cartesian cosmology is based on primordial chaos, which by motion according to fixed laws is ordering to cosmos [1]. According to Descartes the Universe is filled by thin all-penetrable fluid (similar to the ether) which is in a permanent rotational motion. The term vortex itself, "tourbillon", is coming from comparison with turbulent motion of a river. Besides Descartes, the vortex models of gravity were proposed by Bernoulli, Stokes and Huygens. But soon they were displaced by the Newton's gravity theory for a long time. Only in the middle of XIX century the interest to vortex theory revives with works of Helmholtz (1821-1894) [2], Thompson (Lord Kelvin) [3], and Kirchhoff (1824-1887) [4] on the vortex motion of an ideal fluid. The mathematical description of processes related with the motion of vortex in a liquid is starting from Helmholtz's paper "Uber Integrale der Hydrodynamischen Gleichungen Welche den Wirbelbewegungen Entsprechen" (1858) in which he formulated his famous theorem on conservation of vorticity in the rotational motion of a fluid. He also notices an analogy between the fluid motion and the magnetic action of electric fields. General equations of motion for N point vortices (Kirchhoff's equations) have been introduced by Kirchhoff in his lectures in mathematical physics [4]. He derived the corresponding Hamiltonian form of equations and found all possible integrals of the motion. In contrast with Newton's equation for $N$ point masses, having the second order, the Kirchhoff equations are the first order of vortex coordinates. Walter

\footnotetext{
* Corresponding author. Fax: +90 2327507509.

E-mail addresses: nilhangurkan@iyte.edu.tr (Z.N. Gurkan), oktaypashaev@iyte.edu.tr (O. Pashaev).
} 
Gröbli in his thesis "Specille Probleme uber die Bewegung Geradliniger Parallerer Wirbelfaden" in 1877 [5] analyzed the integrable problem of motion of three vortices in the plane. He obtained the system of three nonlinear equations possessing two integrals of motion and allowing to get explicit quadrature. He also considered particular case of the problem of four vortices under condition of symmetry axis and more general problem of $2 N$ vortices with $N$ symmetry axes.

The interest to vortex theory increases with Kelvin's vortex theory of atoms "On Vortex Atoms", [3]. But soon his model was dismissed by quantum mechanical model of atoms [6]. Kelvin also posed the problem of stability under the stationary rotation of the system of $N$ point vortices located at $N$ polygon vertices. He noticed that the problem is similar to the problem of stability for the system of equal magnets floating in external magnetic field. Experiments with floating magnets performed by Mayer, leaded him to the conclusion that for number of vortices (magnets) exceeding 5 , the rotating polygon becomes unstable (in fact the case $N=6$ is stable). The linear stability of polygon has been studied by Thompson. He found that for $n \leqslant 6$ the linear stability takes place, while for $n \geqslant 8$ is not. Stability of the case $n=7$ needs nonlinear analysis and after several failed attempts has been proved only recently. Non-integrability of the four vortex problem in plane, indicating on chaotization of the vortex motion, was found recently [7].

Many problems involving interfacial motion [8] can be cast in the form of vortex sheet dynamics [9]. The discovery of coherent structures in turbulence, increases expectations that the study of vortices will lead to models and an understanding of turbulent flow, one of the great unsolved problems of classical physics. Vortex dynamics is a natural paradigm for the field of chaotic motion and modern dynamical system theory [10]. The theory of line vortices and vortex rings is a part of modern theory of liquid Helium II. Interaction of vortex structures essentially influences on processes in atmosphere and the ocean. In techniques, complete understanding of friction to the motion, noise generation and shock waves, is impossible without clear theory of the vortex motion. In a wide and important class of motions of ideal inviscid fluid [11], the vortex dynamics provides physically profound examples of nonlinear Hamiltonian systems of infinite dimensions, attracting much interest in relation with chaotic phenomena in dynamical systems [12].

Modern applications of vortices extend from liquid crystals and ferromagnets $[13,14]$ to superfluids $[15,16]$ and from non-equilibrium patterns to Quantum Hall effect [17] and cosmic strings [18-20]. They can play essential role in the loop gravity and quantum spacetime, leading to formation of the Cantorian Space [21], fractalization of the microspace geometry, which has been discussed recently in the context of the fractional Quantum Hall Effect $[22,23]$ and Cosmic Strings [24].

Recently it was shown that in ferromagnetic nanomagnets, for particles of various shapes (strips and rings), the switching process involves domain walls which are composite objects made of two or more vortices and edge defects with integer or fractional winding numbers [25]. It may provide a basic model of complex magnetization dynamics in nanomagnets by reducing it to the creation, propagation, and annihilation of a few planar topological defects [26]. The planar vortices represent important class of magnetic systems admitting topologically nontrivial solutions, the dynamics of which is one of the most intriguing questions. Due to nonlinearity in general it is very difficult task to construct exact analytical solution even for one vortex. This is why simplified models admitting exact treatment are important tools to study more realistic situation. In the present paper we consider the simple model of anisotropic planar spin liquid, admitting construction of exact $\mathrm{N}$-vortex and $\mathrm{N}$-vortex lattice solutions with integrable dynamics for arbitrary $\mathrm{N}$.

\section{Topological spin liquid model}

Inspired by superfluid motion at $T=0$ in $\mathrm{He}^{3}-A$, the model introduces the normal velocity of the fermionic liquid as an additional hydrodynamical variable, describing the background fermionic vacuum. The magnetic fluid or spin liquid is characterized by the local magnetization field $\vec{S}(x, y, t)$ subject to the modified Heisenberg model in the moving frame with velocity $\vec{v}(x, y, t)$. Moreover, for planar magnetic systems the existence of topologically nontrivial vortex configurations requires the fluid to be rotational with non-vanishing vorticity function [28]. The system is

$$
\begin{aligned}
& \vec{S}_{t}+v_{1} \partial_{1} \vec{S}-v_{2} \partial_{2} \vec{S}=\vec{S} \times\left(\partial_{1}^{2}-\partial_{2}^{2}\right) \cdot \vec{S}, \\
& \partial_{1} v_{2}-\partial_{2} v_{1}=2 \vec{S}\left(\partial_{1} \vec{S} \times \partial_{2} \cdot \vec{S}\right),
\end{aligned}
$$

where $\vec{S}^{2}(x, y, t)=1$ is classical spin field, $\vec{v}=\left(v_{1}, v_{2}\right)$ is velocity field. The first equation is anisotropic in $x$ and $y$ directions, the second equation, requiring that the fluid vorticity in the plane is proportional to the corresponding magnetic topological current component, is known in the theory of superfluid $\mathrm{He}^{3}$ as the Mermin-Ho relation [15,16]. This system in general should be supplied with the continuity equation $\rho_{t}+\nabla \cdot(\rho \vec{v})=0$ for the density $\rho(x, y, t)$. For incompressible flow $\rho_{t}=0$ the last equation simplifies to $\nabla \cdot \vec{v}=0$ and allows one exclude $\rho$ from consideration. Moreover, for the fluid flow constrained by the incompressibility condition 


$$
\partial_{1} v_{1}+\partial_{2} v_{2}=0
$$

the conservation law

$$
\partial_{t} J_{0}+\partial_{2} J_{2}-\partial_{1} J_{1}=0
$$

holds [35], where

$$
\begin{aligned}
& J_{0}=\left(\partial_{1} \vec{S}\right)^{2}+\left(\partial_{2} \vec{S}\right)^{2}, \\
& J_{1}=-2 \partial_{1} \vec{S} \cdot \vec{S} \times\left(\partial_{1}^{2}-\partial_{2}^{2}\right) \vec{S}+v_{1} J_{0}+2 \vec{S} \cdot\left(\partial_{1} \vec{S} \times \partial_{2}^{2} \vec{S}-\partial_{1} \partial_{2} \vec{S} \times \partial_{2} \vec{S}\right), \\
& J_{2}=2 \partial_{2} \vec{S} \cdot \vec{S} \times\left(\partial_{1}^{2}-\partial_{2}^{2}\right) \vec{S}+v_{2} J_{0}-2 \vec{S} \cdot\left(\partial_{1}^{2} \vec{S} \times \partial_{1} \partial_{2} \vec{S}-\partial_{1} \vec{S} \times \partial_{2} \vec{S}\right) .
\end{aligned}
$$

Due to this, for the incompressible flow (3) the "energy" functional

$$
E=\iint J_{0} \mathrm{~d}^{2} x=\iint\left\{\left(\partial_{1} \vec{S}\right)^{2}+\left(\partial_{2} \vec{S}\right)^{2}\right\} \mathrm{d}^{2} x
$$

is the conserved quantity. The topological charge or the winding number of a spin configuration is defined as

$$
Q=\frac{1}{4 \pi} \iint \vec{S} \cdot\left(\partial_{1} \vec{S} \times \partial_{2} \vec{S}\right) \mathrm{d}^{2} x
$$

and it is also the conserved quantity. These two quantities are related by the Bogomolny type inequality

$$
E \geqslant 8 \pi|Q| \text {. }
$$

It follows from the evident one

$$
\iint\left(\partial_{i} \vec{S} \pm \epsilon_{i j} \vec{S} \times \partial_{j} \vec{S}\right)^{2} \mathrm{~d}^{2} x \geqslant 0
$$

and is saturated by time dependent spin configurations satisfying the self-duality equations

$$
\partial_{i} \vec{S} \pm \epsilon_{i j} \vec{S} \times \partial_{j} \vec{S}=0
$$

If the spin vector phase space, the $2 \mathrm{D}$ sphere, we consider as the Riemann sphere for a complex plane, we can project points on this sphere to that plane by the stereographic projections

$$
S_{+}=S_{1}+\mathrm{i} S_{2}=\frac{2 \zeta}{1+|\zeta|^{2}}, \quad S_{3}=\frac{1-|\zeta|^{2}}{1+|\zeta|^{2}},
$$

where $\zeta(x, y, t)$ is a complex valued function. Now we rewrite the self-duality equation (10) in stereographic projection form. By the complex derivatives, for the first sign in (10) we have the analyticity or the holomorphicity condition:

$$
\zeta_{\bar{z}}(x, y, t)=0
$$

while for the second sign we have the anti-analyticity or the anti-holomorphicity condition:

$$
\zeta_{z}(x, y, t)=0,
$$

where $z=x+\mathrm{i} y$ and $\partial_{z}=\frac{1}{2}\left(\partial_{x}-\mathrm{i} \partial_{y}\right), \partial_{\bar{z}}=\frac{1}{2}\left(\partial_{x}+\mathrm{i} \partial_{y}\right)$. These conditions written in terms of the real $\mathfrak{R} \zeta$ and imaginary $\mathfrak{I}$ parts of function $\zeta$, representing the Cauchy-Riemann equations, describe the incompressible and irrotational fluid flow with the velocity potential $\mathfrak{R} \zeta$ and the stream function $\mathfrak{I} \zeta[27]$.

\section{Anti-holomorphic reduction}

As we can see, analytic/anti-analytic configurations saturate Bogomolny inequality and have minimal energy. This suggests to find solutions of the model (1) with holomorphic/anti-holomorphic stereographic projections. For this reason we first rewrite (1) and (2) in stereographic projection form in complex coordinates $z$ for complex velocities $v_{ \pm}=v_{1} \pm \mathrm{i} v_{2}$ and if $\zeta$ is anti-holomorphic $\zeta_{z}=0$, then the system is reduced to

$$
\begin{aligned}
& \mathrm{i} \zeta_{t}+\mathrm{i} v_{+} \zeta_{\bar{z}}+2 \zeta_{\overline{z z}}-4 \frac{\zeta_{\bar{z}}^{2 \bar{\zeta}}}{1+|\zeta|^{2}}=0, \\
& \partial_{z} v_{+}-\partial_{\bar{z}} v_{-}=-8 \mathrm{i} \frac{\bar{\zeta}_{z} \zeta_{\bar{z}}}{\left(1+|\zeta|^{2}\right)^{2}} .
\end{aligned}
$$


To be consistent, this anti-holomorphicity constraint must be compatible with time evolution. By direct computation we can show that for incompressible flow (3) the anti-holomorphic constraint $\zeta_{z}=0$ is compatible with the time evolution $\partial \zeta_{z} / \partial t=0$. For this flow from (14) and (15) we have $\zeta_{t}+F \zeta_{\bar{z}}=0$ and $F_{z}=0$, where $F \equiv v_{+}-2 \mathrm{i}\left[\ln \frac{\zeta_{\bar{z}}}{\left.1+\mid \xi_{\bar{z}}\right]_{\bar{z}}}\right]_{\bar{z}}$.

\section{Anti-holomorphic Ishimori model}

The above consideration suggests to solve the incompressibility conditions explicitly. So we consider the topological magnet model (1), (2) with incompressibility condition (3). Equation $\vec{\nabla} \cdot \vec{v}=0$ can be solved in terms of the stream function of the flow, $v_{1}=\partial_{2} \psi, v_{2}=-\partial_{1} \psi$, so that we get the so called Ishimori model [29].

$$
\begin{aligned}
& \vec{S}_{t}+\partial_{2} \psi \partial_{1} \vec{S}+\partial_{1} \psi \partial_{2} \vec{S}=\vec{S} \times\left(\partial_{1}^{2} \vec{S}-\partial_{2}^{2} \vec{S}\right), \\
& \left(\partial_{1}^{2}+\partial_{2}^{2}\right) \psi=-2 \vec{S} \cdot\left(\partial_{1} \vec{S} \times \partial_{2} \vec{S}\right) .
\end{aligned}
$$

The Ishimori model is the first example of integrable classical spin model in $2+1$ dimensions [36]. It was shown to be gauge equivalent to the Davey-Stewartson equation, representing the $2+1$ dimensional generalization of the nonlinear Schrödinger equation $[32,33,37]$. Though it was solved in terms of the $\bar{\partial}$ problem, for description of vortices and vortex lattices we propose more simple and elegant method. In terms of complex variables

$$
v_{+}=v_{1}+\mathrm{i} v_{2}=-2 \mathrm{i} \psi_{\bar{z}}, \quad v_{-}=v_{1}-\mathrm{i} v_{2}=2 \mathrm{i} \psi_{z}
$$

for incompressible flow, preserving anti holomorphicity constraint, we have dependence $\zeta=\zeta(\bar{z}, t)$ and the model reduces to the system

$$
\begin{aligned}
& \mathrm{i} \zeta_{t}+2 \psi_{\bar{z}} \zeta_{\bar{z}}+2 \zeta_{\overline{z z}}-\frac{4 \bar{\zeta} \zeta_{\bar{z}}^{2}}{1+|\zeta|^{2}}=0, \\
& \psi_{z \bar{z}}=\frac{2 \bar{\zeta}_{z} \zeta_{\bar{z}}}{\left(1+|\zeta|^{2}\right)^{2}} .
\end{aligned}
$$

We can rearrange the first equation as

$$
\mathrm{i} \zeta_{t}+2 \zeta_{\bar{z}}\left\{\psi-2 \ln \left(1+|\zeta|^{2}\right)+\ln \zeta_{\bar{z}}\right\}_{\bar{z}}=0
$$

If we choose $\psi=2 \ln \left(1+|\zeta|^{2}\right)$, then Eq. (20)

$$
\psi_{z \bar{z}}=2\left[\frac{\bar{\zeta}_{z} \zeta}{1+|\zeta|^{2}}\right]_{\bar{z}}=2 \frac{\bar{\zeta}_{z} \zeta_{\bar{z}}}{\left(1+|\zeta|^{2}\right)^{2}}
$$

is satisfied automatically. Then from (21) for function $\zeta$ we have the anti-holomorphic time dependent Schrödinger equation

$$
i \zeta_{t}+2 \zeta_{\bar{z} \bar{z}}=0 .
$$

By complex analog of the Cole-Hopf transformation $u=4 \zeta_{\bar{z}} / \zeta=4(\log \zeta)_{\bar{z}}$ it implies complex Burgers' equation

$$
\mathrm{i} u_{t}+u u_{\bar{z}}+2 u_{\bar{z} \bar{z}}=0 \text {. }
$$

In (24) $u$ can be interpreted as the complex velocity of effective flow with the complex potential $f(z)=\log \zeta^{4}$. Then every zero of function $\zeta$ corresponds to the vortex solution (pole of complex velocity) of anti-holomorphic Burgers' equation.

\section{Magnetic vortices as moving zeroes of Hermite polynomials}

By stereographic projection (11) at every zero of function $\zeta\left(\bar{z}_{k}, t\right)=0$ we have

$$
\left(S_{1}+\mathrm{i} S_{2}\right)\left(\bar{z}_{k}, t\right)=0, \quad S_{3}\left(\bar{z}_{k}, t\right)=1 .
$$

From another side for the polynomial $\zeta_{N}$ of degree $N$ at infinity $|z| \rightarrow \infty$

$$
\left(S_{1}+\mathrm{i} S_{2}\right)\left(\bar{z}_{k}, t\right)=0, \quad S_{3}\left(\bar{z}_{k}, t\right)=-1 .
$$

It shows that every zero corresponds to the magnetic vortex located at that zero with the spin vector $\vec{S}$ directed up, while at infinity it is directed down (ferromagnetic type order) (see Fig. 1). With such boundary conditions the topological charge $Q$ in (7) is an integer valued and characterizes the number of magnetic vortices. 


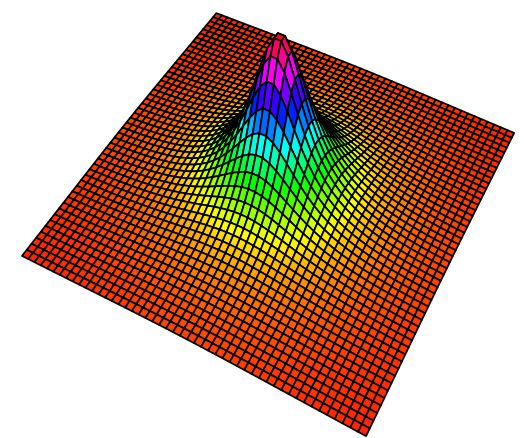

Fig. 1. $N=1$. Magnetic vortex.

If we calculate the topological charge (7) for $N$ zeroes solution

$$
\zeta(\bar{z}, t)=\prod_{k=1}^{N}\left(\bar{z}-\bar{z}_{k}(t)\right)
$$

then we find that $Q=-N$. The above consideration shows that motion of zeroes of time dependent Schrödinger equation (23) describes the motion of magnetic vortices in the Ishimori model. Moreover due to (24) dynamics of these vortices is described by the anti-holomorphic Burgers' equation. To study motion of zeroes of (23) we construct the generating function of the basic vortex solutions by considering complex plane wave solution

$$
\zeta(\bar{z}, t)=\mathrm{e}^{k \bar{z}+2 \mathrm{i}^{2} t} .
$$

Let $x \equiv k \sqrt{\frac{2 t}{\mathrm{i}}}$, then we rewrite it as the generating function for the Hermite polynomials of complex argument

$$
\mathrm{e}^{k \bar{z}+2 i k^{2} t}=\mathrm{e}^{-x^{2}+2\left(\bar{z} \sqrt{\frac{\mathrm{i}}{8 t}}\right) x}=\sum_{n=0}^{\infty} H_{n}\left(\bar{z} \sqrt{\frac{\mathrm{i}}{8 t}}\right) \frac{x^{n}}{n !}
$$

or

$$
\zeta(\bar{z}, t)=\sum_{n=0}^{\infty} \frac{k^{n}}{n !}(-2 \mathrm{i} t)^{n / 2} H_{n}\left(\bar{z} \sqrt{\frac{\mathrm{i}}{8 t}}\right)=\sum_{n=0}^{\infty} \frac{k^{n}}{n !} \Psi_{n}(\bar{z}, t),
$$

where at every power $k^{n}$ we have a polynomial solution of order $n$ :

$$
\Psi_{n}(\bar{z}, t)=\left(\frac{2 t}{\mathrm{i}}\right)^{n / 2} H_{n}\left(\bar{z} \sqrt{\frac{\mathrm{i}}{8 t}}\right) .
$$

This polynomial has $n$ complex roots $\bar{z}_{1}(t), \ldots, \bar{z}_{n}(t)$ describing positions of vortices. For complex zeroes of this function, by identification

$$
\Psi_{N}(\bar{z}, t)=\prod_{k=1}^{N}\left(\bar{z}-\bar{z}_{k}(t)\right)=(-2 \mathrm{i} t)^{N / 2} H_{N}\left(\frac{\bar{z}}{2 \sqrt{-2 \mathrm{i} t}}\right)
$$

we find that $\bar{z}=\bar{z}_{k}(t)$ implies $H_{N}\left(\frac{\bar{z}_{k}}{2 \sqrt{-2 i t}}\right)=0$. Denoting $w_{k}^{(N)}, k=1, \ldots, N$ as zeroes of Hermite polynomials, $H_{N}\left(w_{k}^{(N)}\right)=0$, we have the time dependence for vortex positions

$$
\bar{z}_{k}(t)=2 w_{k}^{(N)} \sqrt{-2 \mathrm{i} t} .
$$

Due to reality of roots $w_{k}^{(N)}$, the form of our solution $\bar{z}_{k}(t)=2 w_{k}^{(N)} \sqrt{-2 \mathrm{i} t}$ implies that all vortices are located on diagonal lines of complex plane:

$$
\bar{z}_{k}(t)=\left|2 w_{k}^{(N)} \sqrt{-2 t}\right| \mathrm{e}^{\mathrm{i} \pi / 4} .
$$

We note that since the time dependence includes square root of time variable $t$, then under time reflection, when $t$ is replaced by $-t$, position of vortices will rotate $\bar{z}_{k} \rightarrow \mathrm{e}^{\mathrm{i} \pi / 2} \bar{z}_{k}$ on angle $\pi / 2$. It means that under collision our vortices change velocity in orthogonal direction and from one diagonal line would be displaced to the orthogonal one. Moreover, sum of 
vortex positions, representing the center of mass of the system, is integral of motion located at the beginning of coordinates.

In Fig. 2 we show contour plot of basic vortex dynamics for four vortices.

In Fig. 3 interaction of two magnetic vortices in Ishimori model is shown.

Since function $\Psi_{N}$ in (32) is solution of the linear equation (23) for any integer $N$, then any linear combination of these functions

$$
\Phi_{N}(\bar{z}, t)=a_{N} \Psi_{N}(\bar{z}, t)+a_{N-1} \Psi_{N-1}(\bar{z}, t)+\cdots+a_{0} \Psi_{0}(\bar{z}, t)
$$

$a_{N} \neq 0$ is also a solution. This solution is determined by $N+1$ complex constants $a_{0}, a_{1}, a_{2}, \ldots, a_{N}$, which are integrals of motion of the system. The higher order coefficient $a_{N} \neq 0$ is not essential and could be put to one. Below we represent particular cases for $N=3$ and $N=4$.

(1) For $N=3$ we have the general solution

$$
\Phi(\bar{z}, t)=\left(\bar{z}^{3}+12 \bar{z} \mathrm{i} t\right)+a_{2}\left(\bar{z}^{2}+4 \mathrm{i} t\right)+a_{1} \bar{z}+a_{0} .
$$

This cubic in $z$ equation has three complex roots $\bar{z}_{1}(t), \bar{z}_{2}(t), \bar{z}_{3}(t)$ moving in plane according to the systems (76). Instead of solving that system of differential equations we will find roots of cubic equation according Cardano formulas. Coefficient

$$
-a_{2}=\bar{z}_{1}(t)+\bar{z}_{2}(t)+\bar{z}_{3}(t)
$$

is integral of motion having meaning of the center of mass for three vortices. Without loss of generality we can always choose coordinate system with the beginning at this center of mass. So we will put $a_{2}=0$. Then our cubic equation has the reduced Cardano form

$$
\bar{z}^{3}+p \bar{z}+q=0,
$$

where $p=a_{1}+12 \bar{z}$ it, $q=a_{0}+4 a_{2}$ it. Solution of this equation is

$$
\bar{z}_{1}=\alpha_{1}+\beta_{1}, \quad \bar{z}_{2}=\alpha_{1} \omega_{1}+\beta_{1} \omega_{2}, \quad \bar{z}_{3}=\alpha_{1} \omega_{2}+\beta_{1} \omega_{1}
$$
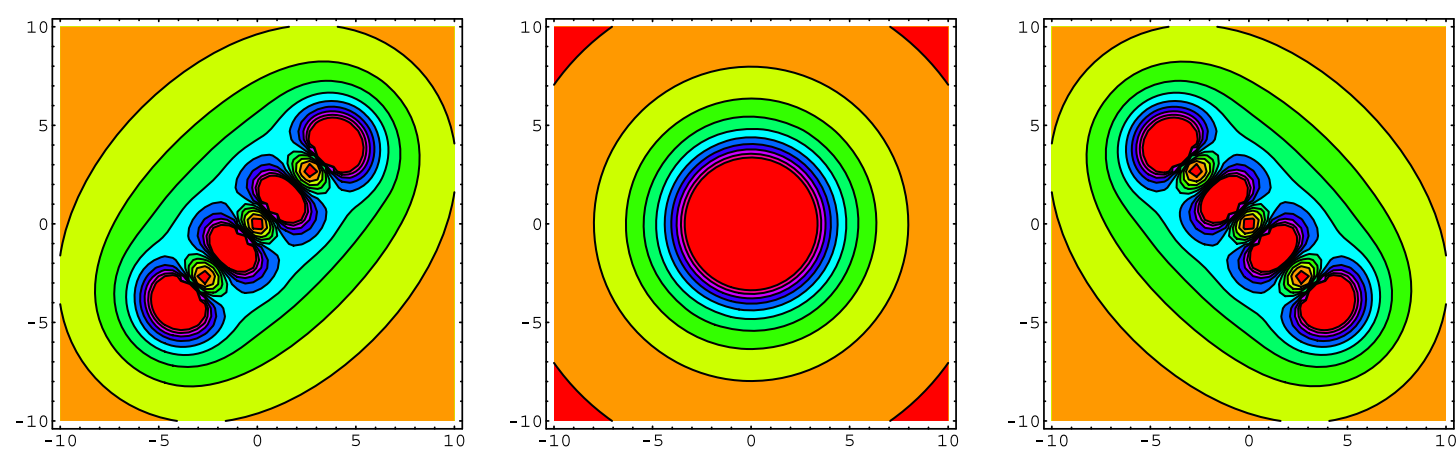

Fig. 2. $N=4$. Vortex dynamics.
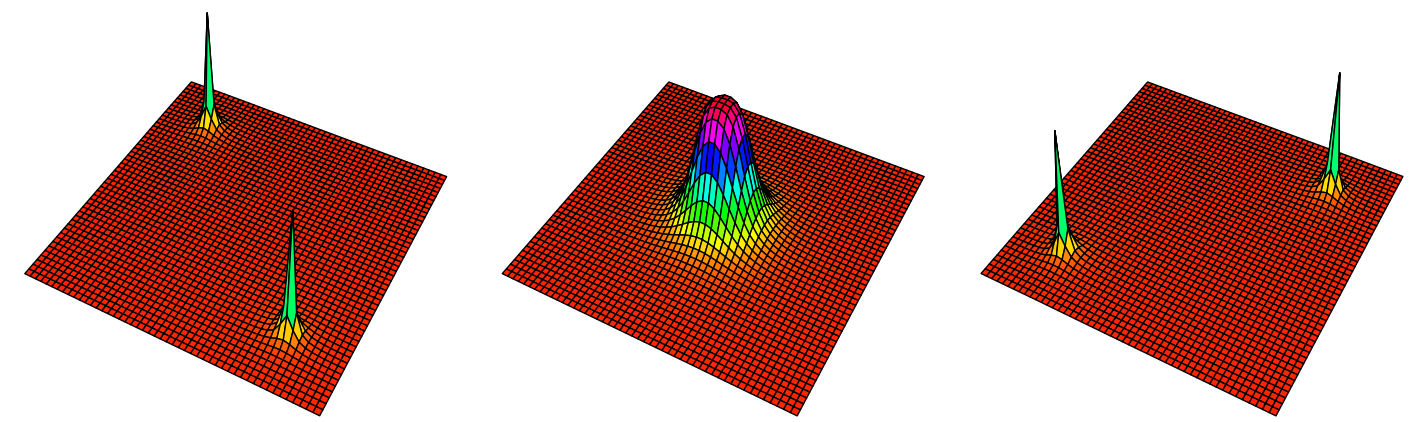

Fig. 3. $N=2$. Magnetic vortex dynamics. 
where $\alpha_{1}, \beta_{1}$ is one of the couple of roots

$$
\alpha=\left(-\frac{q}{2}+\sqrt{\frac{q^{2}}{4}+\frac{p^{3}}{27}}\right)^{1 / 3} \quad \beta=\left(-\frac{q}{2}-\sqrt{\frac{q^{2}}{4}+\frac{p^{3}}{27}}\right)^{1 / 3}
$$

and

$$
\omega_{1}=-1 / 2+\mathrm{i} \sqrt{3} / 2=\mathrm{e}^{\mathrm{i} 2 \pi / 3}, \quad \omega_{2}=-1 / 2-\mathrm{i} \sqrt{3} / 2=\mathrm{e}^{-\mathrm{i} 2 \pi / 3}
$$

are cubic roots of 1 . For particular values $a_{0}=a_{2}=0$ when $q=0$ our roots become

$$
\begin{aligned}
& \bar{z}_{1}=\sqrt{4 \mathrm{i} t}\left(1+\mathrm{e}^{\mathrm{i} \pi / 3}\right), \\
& \bar{z}_{2}=\sqrt{4 \mathrm{i} t}\left(\omega_{1}+\omega_{2} \mathrm{e}^{\mathrm{i} \pi / 3}\right), \\
& \bar{z}_{3}=\sqrt{4 \mathrm{i} t}\left(\omega_{2}+\omega_{1} \mathrm{e}^{\mathrm{i} \pi / 3}\right)
\end{aligned}
$$

and coincide with particular cases (33) when one of the vortices, $\bar{z}_{2}=0$, is static at the beginning of coordinates. In general case due to non-vanishing orbital momentum no one of three vortices crosses beginning of coordinates.

(2) For $N=4$ case we have

$$
\Phi(\bar{z}, t)=\left(\bar{z}^{4}+24 \bar{z}^{2} \mathrm{i} t+12(-2 \mathrm{i} t)^{2}\right)+a_{3}\left(\bar{z}^{3}+12 \bar{z} \mathrm{i} t\right)+a_{2}\left(\bar{z}^{2}+4 \mathrm{i} t\right)+a_{1} \bar{z}+a_{0}
$$

and like in previous case we choose the center of mass at the beginning of coordinate system, so that the coefficient

$$
-a_{3}=\bar{z}_{1}(t)+\bar{z}_{2}(t)+\bar{z}_{3}(t)+\bar{z}_{4}(t)=0 .
$$

Explicit form of the roots in this case can be done in radicals. Below we will write them only for special case $a_{1}=0$. Solving bi-quadratic equation $\bar{z}^{4}+\bar{z}^{2}\left[a_{2}+24 \mathrm{i} t\right]+\left[a_{0}+4 a_{2} \mathrm{i} t+12(-2 \mathrm{i} t)^{2}\right]=0$ we have four roots:

$$
\begin{aligned}
& \bar{z}_{1,2}= \pm \sqrt{-\left[\frac{a_{2}}{2}+12 \mathrm{i} t\right]+\sqrt{\left(\frac{a_{2}}{2}+12 \mathrm{i} t\right)^{2}-\left[a_{0}+4 a_{2} \mathrm{i} t+12(-2 \mathrm{i} t)^{2}\right]}}, \\
& \bar{z}_{3,4}= \pm \sqrt{-\left[\frac{a_{2}}{2}+12 \mathrm{i} t\right]-\sqrt{\left(\frac{a_{2}}{2}+12 \mathrm{i} t\right)^{2}-\left[a_{0}+4 a_{2} \mathrm{i} t+12(-2 \mathrm{i} t)^{2}\right]}} .
\end{aligned}
$$

From dynamics of 3 and 4 vortices we can see that differences appear for the vortex motion at finite times. But for large time the behavior of vortices is similar to the particular case (33). Explanation of this fact can be done for general $\mathrm{N}$-vortex configuration. Indeed, if we consider asymptotic form of the general $\mathrm{N}$-vortex solution (35), when $t \rightarrow \infty$ and $\bar{z} \rightarrow \infty$ such that $|z|^{2} / t \rightarrow$ const, then we can see that all terms of the function $\Psi_{N}$ for any $N$ have the same order. Moreover, the dominant role in (35) plays the function $\Psi_{N}$ with highest order of $N$. But it is exactly solution (32) which we found before. So we proved that asymptotically our vortices will follow diagonal lines according to the low (33): $\bar{z}_{k}(t)=2 w_{k} \sqrt{-2 \mathrm{i} t}$. If we calculate complex velocity corresponding to $k$-vortex $u_{k}=\mathrm{d} \bar{z}_{k} / \mathrm{d} t=w_{k} \sqrt{-2 \mathrm{i} / t}$ then at large times $t \rightarrow \infty$ the velocity of the vortex is decreasing up to zero $u_{k} \rightarrow 0$, as the inverse square root of time $\sim 1 / \sqrt{t}$.

\section{Vortex solutions generating technique}

In this section we propose a general method allowing us to create an arbitrary number of vortices on given background solution. In general, $N$ vortex configuration is described by complex polynomial function degree $N$ where coefficients are represented in terms of symmetric polynomials. The polynomial $P_{1}=\bar{z}_{1}+\cdots+\bar{z}_{N}$ is integral of motion having meaning of the center of mass for $N$ vortices. However, all other polynomials are not integrals of motion, only proper combinations of these polynomials provide integrals of motion of the system. Moreover, to find solutions $\bar{z}_{1}(t), \ldots, \bar{z}_{N}(t)$ in terms of these polynomials means solving algebraic equation degree $N$, which as known to be solvable in radicals only for $N \leqslant 4$. This is why to add a new zero or vortex to the system we follow another approach. In Section 4 we have constructed basic solutions in terms of Hermite polynomials of complex argument (32). Adding recurrence relations

$$
\begin{aligned}
& H_{N+1}(x)=2 x H_{N}(x)-2 N H_{N-1}(x), \\
& 0=-H_{N}^{\prime}(x)+2 N H_{N-1}(x)
\end{aligned}
$$

one can get 


$$
H_{N+1}(x)=\left(2 x-\frac{\mathrm{d}}{\mathrm{d} x}\right) H_{N}(x) .
$$

Recursively it leads to following operator representation of the standard Hermite polynomials [38]

$$
H_{N}(x)=\left(2 x-\frac{\mathrm{d}}{\mathrm{d} x}\right)^{N} \cdot 1
$$

which implies for complex argument

$$
\Psi_{N}(\bar{z}, t)=(-2 \mathrm{i} t)^{N / 2}\left(\frac{\bar{z}}{\sqrt{-2 \mathrm{i} t}}-2 \sqrt{-2 \mathrm{i} t} \frac{\partial}{\partial \bar{z}}\right)^{N} \cdot 1
$$

and we have operator representation for our basic solution (32)

$$
\Psi_{N}(\bar{z}, t)=\left(\bar{z}+4 \mathrm{i} t \frac{\partial}{\partial \bar{z}}\right)^{N} \cdot 1
$$

This representation suggests the form of generating operator for solutions of our Eq. (23). By direct substitution we can prove the next, vortex generation technique for solutions of this equation. If $\Phi(\bar{z}, t)$ is a solution of $(23)$, then function

$$
\Psi(\bar{z}, t)=\left(\bar{z}+4 \mathrm{i} t \frac{\partial}{\partial \bar{z}}\right) \Phi(\bar{z}, t)
$$

is also a solution of (23). This solution add one vortex to the background configuration $\Phi(\bar{z}, t)$. Applying it several times one can add arbitrary number of vortices. Eq. (23) has evident solution $\Phi=1$. Then

$$
\Psi_{1}(\bar{z}, t)=\left(\bar{z}+4 \mathrm{i} t \frac{\partial}{\partial \bar{z}}\right) \Phi(\bar{z}, t)=\left(\bar{z}+4 \mathrm{i} t \frac{\partial}{\partial \bar{z}}\right) \cdot 1
$$

is also a solution of (23). Next we have

$$
\Psi_{2}(\bar{z}, t)=\left(\bar{z}+4 \mathrm{i} t \frac{\partial}{\partial \bar{z}}\right) \Psi_{1}=\left(\bar{z}+4 \mathrm{i} t \frac{\partial}{\partial \bar{z}}\right)^{2} \cdot 1 .
$$

Continuing this procedure we have solution of (23) for an arbitrary positive integer $N$

$$
\Psi_{N}(\bar{z}, t)=\left(\bar{z}+4 \mathrm{i} t \frac{\partial}{\partial \bar{z}}\right) \Psi_{N-1}=\cdots=\left(\bar{z}+4 \mathrm{i} t \frac{\partial}{\partial \bar{z}}\right)^{N} \cdot 1 .
$$

This way we derived particular operator representation for $N$ vortex solution

$$
\Psi_{N}(\bar{z}, t)=\prod_{i=1}^{N}\left(\bar{z}-\bar{z}_{i}(t)\right)=\left(\bar{z}+4 \mathrm{i} t \frac{\partial}{\partial \bar{z}}\right)^{N} 1 .
$$

Using previous result and linearity of (23) we have the next generalization. If $\Phi(\bar{z}, t)$ is a solution of Eq. (23) then function

$$
\Psi(\bar{z}, t)=\sum_{n=0}^{N} a_{n}\left(\bar{z}+4 \mathrm{i} t \frac{\mathrm{d}}{\mathrm{d} \bar{z}}\right)^{n} \Phi(\bar{z}, t),
$$

where $a_{0}, \ldots, a_{N}$ are arbitrary constants, is also a solution of Eq. (23). As easy to see adding to the system a new vortex in a proper way, we add an additional integral of motion. Let us suppose that we have solution $\Psi(\bar{z}, t)$ with $N$-simple zeroes at points $\bar{z}_{1}, \ldots, \bar{z}_{N}$, that means, $\Psi\left(\bar{z}_{n}, t\right)=0, n=1, \ldots, N$. Then due to

$$
\Psi(\bar{z}, t)=\prod_{n=1}^{N}\left(\bar{z}-\bar{z}_{n}(t)\right)=\sum_{n=0}^{N} a_{n} \Psi_{n}(\bar{z}, t)
$$

we have the system of $N$ linear algebraic equations $\sum_{n=0}^{N} a_{n} \Psi_{n}\left(\bar{z}_{k}, t\right)=0, k=1, \ldots, N$. Extracting $n=0$ term and dividing on $a_{0}$ it can be rewritten in the form of inhomogeneous system of $N$ algebraic equations

$$
\sum_{n=1}^{N} b_{n} \Psi_{n}\left(\bar{z}_{k}, t\right)=-1, \quad k=1, \ldots, N
$$

on $N$ variables $b_{n}=a_{n} / a_{0}$. Then, $N$ integrals of motion can be found by Crammers formulas $b_{k}=\Delta_{k} / \Delta, k=1, \ldots, N$. From the above consideration if we have $\Phi(\bar{z}, t)$ as a solution of Eq. (23) and $F(\bar{z})$ is anti-analytic function in some domain $D_{0}=\{|z|<R\}$, then function 


$$
\Psi(\bar{z}, t)=F\left(\bar{z}+4 \mathrm{i} t \frac{\mathrm{d}}{\mathrm{d} \bar{z}}\right) \Phi(\bar{z}, t)
$$

is also a solution of Eq. (23). Since coefficients $a_{n} \equiv \frac{F^{(n)}(0)}{n !}$ are integrals of motion, the function $F$ can be considered as the generating function of integrals of motion. At the end of this section we note that operator $K=\bar{z}+4 \mathrm{i} t \partial_{\bar{z}}$ in our approach is commuting with the Schrödinger operator $S=i \partial_{t}+2 \partial_{\bar{z}}^{2}$ and represent the complex boost transformation of the Galilean group.

\section{Sine-Hermite solution and stationary vortex lattice}

As an application let us consider entire function

$$
F(w)=\sin w=\sum_{n=0}^{\infty}(-1)^{n} \frac{w^{2 n+1}}{(2 n+1) !} .
$$

Then according to the above consideration

$$
\Phi(\bar{z}, t)=\sum_{n=0}^{\infty} \frac{(-1)^{n}}{(2 n+1) !}\left(\bar{z}+4 \mathrm{i} t \frac{\partial}{\partial \bar{z}}\right)^{2 n+1} \cdot 1
$$

is solution of Eq. (23). According to (51) we rewrite it as

$$
\Phi(\bar{z}, t)=\sum_{n=0}^{\infty} \frac{(-1)^{n}}{(2 n+1) !} \Psi_{2 n+1}(\bar{z}, t)=\Psi_{1}(\bar{z}, t)-\frac{\Psi_{3}(\bar{z}, t)}{3 !}+\frac{\Psi_{5}(\bar{z}, t)}{5 !}-\cdots
$$

or by (32)

$$
\begin{aligned}
\Phi(\bar{z}, t) & =\sum_{n=0}^{\infty} \frac{(-1)^{n}}{(2 n+1) !}(-2 \mathrm{i} t)^{n+\frac{1}{2}} H_{2 n+1}\left(\frac{\bar{z}}{2 \sqrt{-2 \mathrm{i} t}}\right) \\
& =(-2 \mathrm{i} t)^{n+\frac{1}{2}} H_{1}\left(\frac{\bar{z}}{2 \sqrt{-2 \mathrm{i} t}}\right)-\frac{1}{3 !}(-2 \mathrm{i} t)^{n+\frac{3}{2}} H_{3}\left(\frac{\bar{z}}{2 \sqrt{-2 \mathrm{i} t}}\right)+\frac{1}{5 !}(-2 \mathrm{i} t)^{n+\frac{5}{2}} H_{5}\left(\frac{\bar{z}}{2 \sqrt{-2 \mathrm{i} t}}\right)-\cdots
\end{aligned}
$$

Writing operator

$$
\sin \left(\bar{z}+4 \mathrm{i} t \frac{\partial}{\partial \bar{z}}\right)=\frac{1}{2 \mathrm{i}}\left(\exp \left[\mathrm{i}\left(\bar{z}+4 \mathrm{i} t \frac{\partial}{\partial \bar{z}}\right)\right]-\exp \left[-\mathrm{i}\left(\bar{z}+4 \mathrm{i} t \frac{\partial}{\partial \bar{z}}\right)\right]\right)
$$

and using Baker-Hausdorff relation for non-commuting operators $\bar{z}$, $\frac{\partial}{\partial \bar{z}}$ we have

$$
\sin \left(\bar{z}+4 \mathrm{i} t \frac{\partial}{\partial \bar{z}}\right)=-\frac{\mathrm{i}}{2} \mathrm{e}^{-2 \mathrm{i} t}\left(\mathrm{e}^{\mathrm{i} \overline{\bar{z}}} \mathrm{e}^{-4 t \partial / \partial \bar{z}}-\mathrm{e}^{-\mathrm{i} \bar{z}} \mathrm{e}^{4 t \partial / \partial \bar{z}}\right)
$$

or

$$
\sin \left(\bar{z}+4 \mathrm{i} t \frac{\partial}{\partial \bar{z}}\right)=\mathrm{ie}^{-2 \mathrm{i} t}\left[\cos \bar{z} \sinh \left(4 t \frac{\partial}{\partial \bar{z}}\right)-\mathrm{i} \sin \bar{z} \cosh \left(4 t \frac{\partial}{\partial \bar{z}}\right)\right] .
$$

Applying this operator to evident solution $\Psi=1$ we get

$$
\sin \left(\bar{z}+4 \mathrm{i} t \frac{\partial}{\partial \bar{z}}\right) \cdot 1=\mathrm{e}^{-2 i t} \sin \bar{z}
$$

It describes the time dependent stationary vortex lattice solution of (23). Positions of vortices in the lattice do not change with time. Comparing with (62) we have the next expansion in terms of Hermite polynomials

$$
\begin{aligned}
& \exp (-2 \mathrm{i} t) \sin \bar{z}=(-2 \mathrm{i} t)^{n+\frac{1}{2}} H_{1}\left(\frac{\bar{z}}{2 \sqrt{-2 \mathrm{i} t}}\right) \\
& -\frac{1}{3 !}(-2 \mathrm{i} t)^{n+\frac{3}{2}} H_{3}\left(\frac{\bar{z}}{2 \sqrt{-2 \mathrm{i} t}}\right)+\frac{1}{5 !}(-2 \mathrm{i} t)^{n+\frac{5}{2}} H_{5}\left(\frac{\bar{z}}{2 \sqrt{-2 \mathrm{i} t}}\right)-\cdots
\end{aligned}
$$

Then in terms of $\Psi_{n}$ it gives

$$
\Phi(\bar{z}, t)=\mathrm{e}^{-2 \mathrm{i} t} \sin \bar{z}=\Psi_{1}(\bar{z}, t)-\frac{\Psi_{3}(\bar{z}, t)}{3 !}+\frac{\Psi_{5}(\bar{z}, t)}{5 !}-\cdots
$$


The last formula describes decomposition of the stationary periodic lattice in terms of motion of odd $1,3,5, \ldots, 2 n+1, \ldots$ number of vortices. It shows that proper superposition of odd number basic vortex motions described in Section 5 leads to the fixed in time periodic lattice of vortices (vortex crystal).

\section{Single vortex-vortex lattice collision}

As another application of the results from Section 5, now we construct a new class of solutions describing single vortex collision with the vortex chain lattices. Consider solution of (23) in the double lattice form

$$
\zeta(\bar{z}, t)=\mathrm{e}^{-8 i t} \sin \left(\bar{z}-\bar{z}_{1}(t)\right) \sin \left(\bar{z}+\bar{z}_{1}(t)\right), \quad \cos 2 \bar{z}_{1}(t)=r \mathrm{e}^{8 i t}
$$

then we have another solution of (23) in the form

$$
\Psi(\bar{z}, t)=\left(\bar{z}+4 \mathrm{i} t \frac{\partial}{\partial \bar{z}}\right) \frac{1}{2}\left(r-\mathrm{e}^{-8 \mathrm{it}} \cos 2 \bar{z}\right)=\frac{1}{2}\left[r \mathrm{e}^{8 \mathrm{iit}} \bar{z}-\bar{z} \cos 2 \bar{z}+8 \mathrm{i} t \sin 2 \bar{z}\right] \mathrm{e}^{-8 i t} .
$$

Using properties of trigonometric function of complex argument

$$
\sin 2 \bar{z}=\sin 2 x \cosh 2 y-\mathrm{i} \cos 2 x \sinh 2 y
$$

we have for the real and imaginary parts of function $\Psi$ following expressions correspondingly

$$
\begin{aligned}
& \mathfrak{R} \Psi=\frac{r}{2} x \cos 8 t+\frac{r}{2} y \sin 8 t+8 t \cos 2 x \sinh 2 y-\frac{1}{2} x \cos 2 x \cosh 2 y-\frac{1}{2} y \sin 2 x \sinh 2 y, \\
& \Im \Psi=\frac{r}{2} x \sin 8 t-\frac{r}{2} y \cos 8 t+8 t \sin 2 x \cosh 2 y+\frac{1}{2} y \cos 2 x \cosh 2 y-\frac{1}{2} x \sin 2 x \sinh 2 y .
\end{aligned}
$$

In Fig. 4 we show collision of a single vortex with the double vortex lattices at positive time $t>0$. As we can see, addition of the vortex leads to dimerization of the lattice vortices which propagates in both directions and creates finally the dimerized lattice.

Following the same idea we can consider solution describing interaction of $N$-vortices with $M$-vortex chain lattices (vortex crystal) in the form

$$
\Psi(\bar{z}, t)=\mathrm{e}^{-2 \mathrm{i} M^{2} t}\left(\bar{z}+4 \mathrm{i} t \frac{\partial}{\partial \bar{z}}\right)^{N} \prod_{k=1}^{M} \sin \left(\bar{z}-\bar{z}_{k}(t)\right) .
$$

\section{Integrable $N$-particle problem for $N$-vortex motion}

In this section we show that problem of $N$-point vortices in the plane can be reduced to complexified version of the Calogero-Moser model type I [30,31]. As we have seen in Section 4 the system of $N$ point vortices is described by function (27) satisfying the anti-holomorphic Schrödinger equation (23). Then positions of vortices in the complex plane, $\bar{z}_{1}, \ldots, \bar{z}_{N}$, are subject to the first order system

$$
\frac{\mathrm{d}}{\mathrm{d} t} \bar{z}_{j}=-4 \mathrm{i} \sum_{k \neq(j)}^{N} \frac{1}{\left(\bar{z}_{j}-\bar{z}_{k}\right)} .
$$

In one space dimension this system has been considered first in [30,39] for moving poles of Burgers' equation, determined by zeroes of the heat equation. However, complexification of the problem has several advantages [42]. First of all the root problem of algebraic equation degree $N$ is complete in the complex domain as well as the moving singularity analysis of differential equations. In contrast to the one dimension, the pole dynamics in the plane becomes time reversible (see discussion after Eq. (34)) and has interpretation of the vortex dynamics. If we differentiate (76) once and use the system again then we have the second order Newton's equations of motion

$$
\frac{\mathrm{d}^{2}}{\mathrm{~d} t^{2}} \bar{z}_{j}=\sum_{k \neq(j)}^{N} \frac{16}{\left(\bar{z}_{j}-\bar{z}_{k}\right)^{3}} .
$$

These equations have Hamiltonian form 

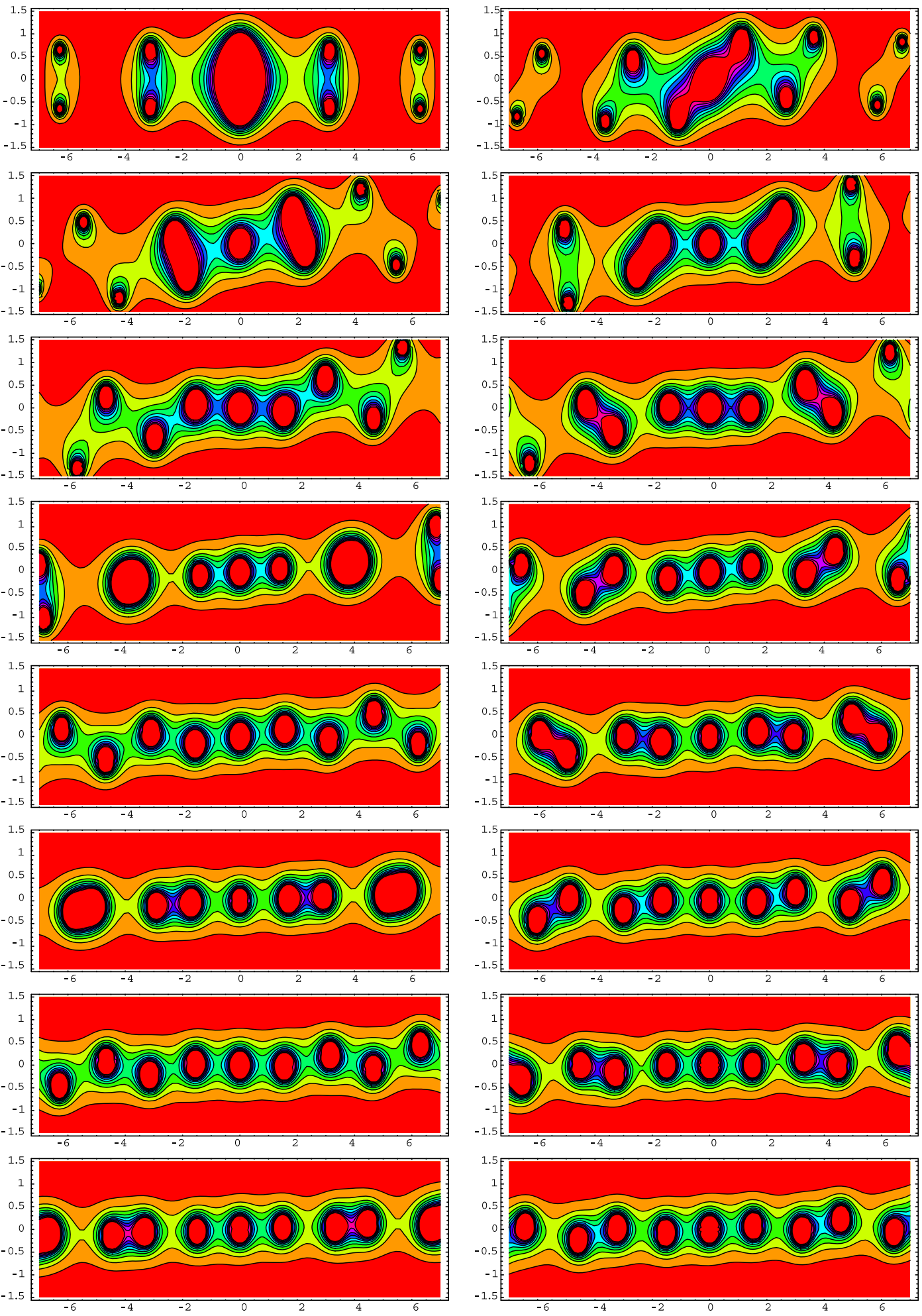

Fig. 4. Single vortex -2 vortex lattice dynamics. 


$$
\dot{\bar{z}}_{j}=\frac{\partial H}{\partial p_{j}}=p_{j}, \quad \dot{p}=-\frac{\partial H}{\partial \bar{z}_{j}}
$$

with Hamiltonian function

$$
H=\frac{1}{2} \sum_{j=1}^{N} p_{j}^{2}+\sum_{j<k} \frac{8}{\left(\bar{z}_{j}-\bar{z}_{k}\right)^{2}} .
$$

The system (77) is complexified version of the Calogero-Moser system type I, where $N$-particle positions, $q_{1}, \ldots, q_{N}$ should be replaced by complex vortex positions $\bar{z}_{1}, \ldots, \bar{z}_{N}$. The Hamiltonian equation (78) are equivalent to the Lax matrix equation

$$
\mathrm{i} \dot{L}=A L-L A
$$

where

$$
\begin{aligned}
L_{j k} & =\delta_{j k} p_{j}+\mathrm{i} g\left(1-\delta_{j k}\right) \frac{1}{\bar{z}_{j}-\bar{z}_{k}} \\
A_{j k} & =g\left[\delta_{j k} \sum_{l \neq j} \frac{1}{\left(\bar{z}_{j}-\bar{z}_{l}\right)^{2}}-\left(1-\delta_{j k}\right) \frac{1}{\left(\bar{z}_{j}-\bar{z}_{k}\right)^{2}}\right],
\end{aligned}
$$

and the coupling constant $g=\sqrt{-4}$. Since matrix $L(t)$ is isospectrally deformed with time, the corresponding (complex) eigenvalues are time independent integrals of motion. Their symmetric functions as integrals of motion are given by $I_{k}=\operatorname{tr} L^{k+1}$. It shows that complexified Calogero-Moser system is an integrable system and as a consequence, the $\mathrm{N}$-vortex system (76), which has been mapped to Calogero-Moser system, is also integrable.

\section{Integrable $N$-particle problem for $N$-vortex crystals}

Similarly to the previous case now we consider mapping of the $N$-vortex chain lattices (vortex crystals) to the complexified Calogero-Moser system of types II and III [31]. For simplicity first we consider the system of two vortex chain lattices described by function

$$
\zeta(\bar{z}, t)=\mathrm{e}^{-4 \mathrm{i} t} \sin \left(\bar{z}-\bar{z}_{1}(t)\right) \sin \left(\bar{z}-\bar{z}_{2}(t)\right)
$$

so that position of lattices is subject to the first order system

$$
\dot{\bar{z}}_{1}=-4 \mathrm{i} \cot \left(\bar{z}_{1}-\bar{z}_{2}\right), \quad \dot{\bar{z}}_{2}=4 \mathrm{i} \cot \left(\bar{z}_{1}-\bar{z}_{2}\right) \text {. }
$$

Differentiating once in time we get Newton's equations in the Hamiltonian form

$$
\begin{aligned}
& \dot{\bar{z}}_{1}=\frac{\partial H}{\partial p_{1}}=p_{1}, \quad \dot{p}_{1}=-\frac{\partial H}{\partial \bar{z}_{1}}=32 \frac{\cot \left(\bar{z}_{1}-\bar{z}_{2}\right)}{\sin ^{3}\left(\bar{z}_{1}-\bar{z}_{2}\right)}, \\
& \dot{\bar{z}}_{2}=\frac{\partial H}{\partial p_{2}}=p_{2}, \quad \dot{p}_{2}=-\frac{\partial H}{\partial \bar{z}_{2}}=32 \frac{\cot \left(\bar{z}_{2}-\bar{z}_{1}\right)}{\sin ^{3}\left(\bar{z}_{2}-\bar{z}_{1}\right)}
\end{aligned}
$$

with Hamiltonian function

$$
H=\frac{p_{1}^{2}}{2}+\frac{p_{2}^{2}}{2}+\frac{16}{\sin ^{2}\left(\bar{z}_{1}-\bar{z}_{2}\right)} .
$$

Comparing this Hamiltonian of two vortex lattices with the Calogero-Moser system, we realize that it corresponds to complexified version of the model type III. We can generalize this result considering $N$ vortex chain lattices in the horizontal direction $x$. Positions of lattices are subject to the first order system

$$
\dot{\bar{z}}_{j}=-4 \mathrm{i} \sum_{k=1,(k \neq j)}^{N} \cot \left(\bar{z}_{j}-\bar{z}_{k}\right), \quad j=1, \ldots, N .
$$

Differentiating once we get

$$
\ddot{\bar{z}}_{j}=-32 \sum_{k=1,(k \neq j)}^{N} \frac{\cot \left(\bar{z}_{j}-\bar{z}_{k}\right)}{\sin ^{2}\left(\bar{z}_{j}-\bar{z}_{k}\right)}, \quad j=1, \ldots, N
$$


which is complexified Calogero-Moser system type III with Hamiltonian

$$
H=\frac{1}{2} \sum_{j} p_{j}^{2}+\sum_{j<k} \frac{16}{\sin ^{2}\left(\bar{z}_{j}-\bar{z}_{k}\right)} .
$$

If instead of horizontal $x$ direction, we consider $N$ chain lattices in the vertical $y$ direction, it results in rotation of every zero on angle $\pi / 2$ and the replacement of complex function $\sin \bar{z}$ by $\sinh \bar{z}$. As a result, the corresponding Calagero-Moser system would be of type II. This consideration shows also equivalence of complexified Calogero-Moser systems of types II and III.

\section{Time dependent Schrödinger problem in harmonic potential}

Vorticity equation (22) is invariant under substitution $\psi \rightarrow \psi+U$ where $U$ is an arbitrary harmonic function: $\Delta U=0$. Choosing

$$
\psi=2 \ln \left(1+|\zeta|^{2}\right)+U(\bar{z}, t)+\bar{U}(z, t)
$$

and substituting to (21) we have complex Schrödinger equation with additional potential term

$$
\mathrm{i} \zeta_{t}+\zeta_{\overline{z z}}+\zeta_{\bar{z}} U_{\bar{z}}=0 .
$$

\section{Bound state of vortices}

Here we choose particular form $U(\bar{z}, t)=\frac{1}{2} \bar{z}^{2}$ so that

$$
\psi=2 \ln \left(1+|\zeta|^{2}\right)+\frac{1}{2}\left(\bar{z}^{2}+z^{2}\right) .
$$

Then we have time evolution subject to the equation

$$
i \zeta_{t}+2 \zeta_{\overline{z z}}+\bar{z} \zeta_{\bar{z}}=0 \text {. }
$$

Looking for solution in the form $[30,42]$

$$
\zeta(\bar{z}, t)=\sum_{n} \mathrm{e}^{\mathrm{i} n t} u_{n}(\bar{z})
$$

we find that function $u_{n}(\bar{z})$ satisfies the complex Hermite equation

$$
u_{n}^{\prime \prime}+\bar{z} u_{n}^{\prime}+u_{n}=0 .
$$

It gives time dependent vortex solution in the form

$$
\zeta(\bar{z}, t)=\sum_{n=0}^{N} \mathrm{e}^{\mathrm{i} n t} H_{n}(\bar{z}) .
$$

This solution is $N$ th degree polynomial with periodic time dependent coefficients and it describes the bound state of $N$ magnetic vortices. For particular value $N=2$ we have

$$
\begin{aligned}
& \zeta(\bar{z}, t)=H_{0}(\bar{z})+\mathrm{e}^{\mathrm{i} t} H_{1}(\bar{z})+\mathrm{e}^{2 \mathrm{it} t} H_{2}(\bar{z}), \\
& \mathfrak{R} \zeta=1+2 x \cos t+2 y \sin t+\left[4\left(x^{2}-y^{2}\right)-2\right] \cos 2 t+8 x y \sin 2 t, \\
& \mathfrak{\Im} \zeta=-2 y \cos t+2 x \sin t-8 x y \cos 2 t+\left[4\left(x^{2}-y^{2}\right)-2\right] \sin 2 t .
\end{aligned}
$$

This solution is periodic in time with period $T=2 \pi$ and it describes the bound state of two magnetic vortices (see Fig. 5).

\section{Static vortex crystals and the Liouville equation}

From previous considerations we have seen that static vortex configurations for Eq. (23) are exist only for $N=1$. One can ask if there exist static $N$ vortex or even $N$-vortex lattices-vortex crystals for the Ishimori model (16) and (17). To answer this question we return back to (21). For static configurations $\partial \zeta / \partial t=0$ implies 

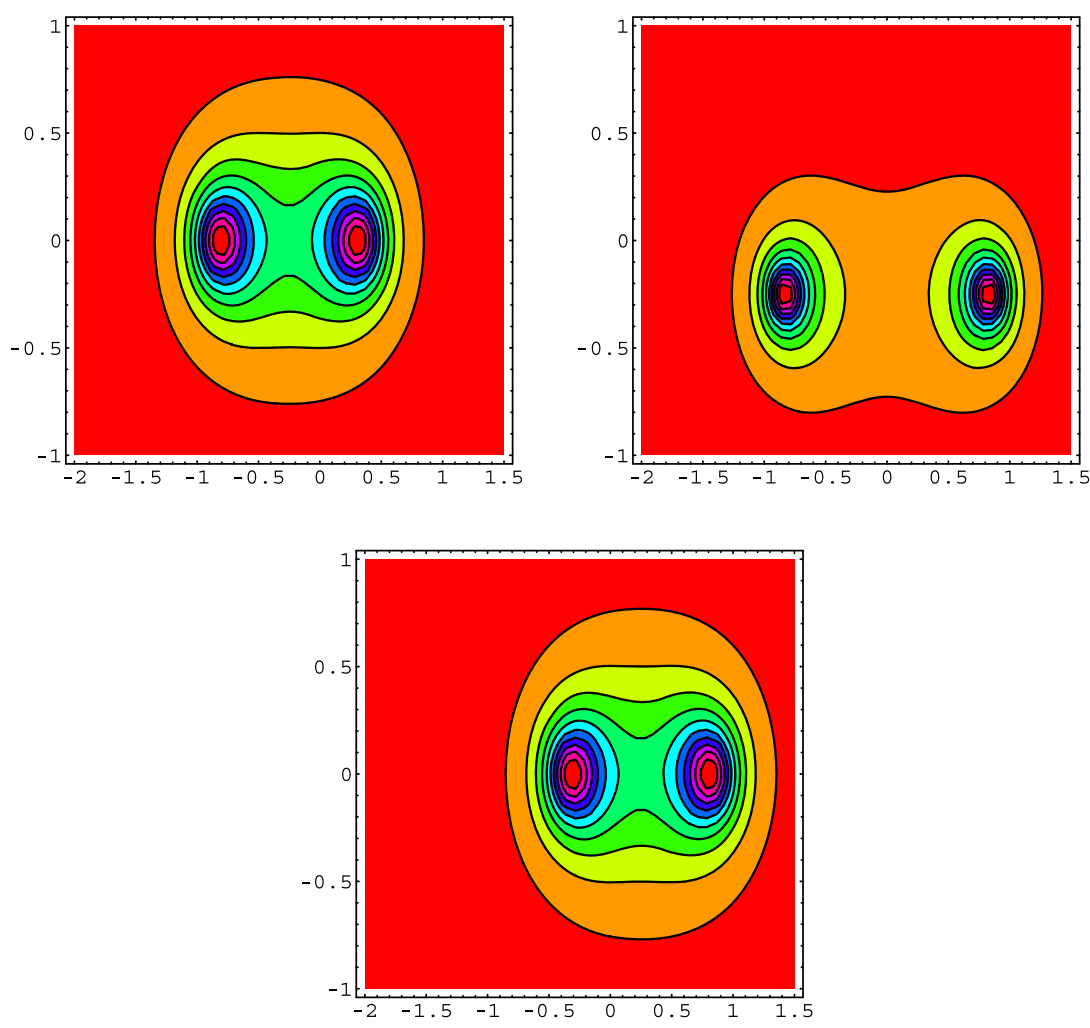

Fig. 5. Bound state of two magnetic vortices.

$$
\psi=2 \ln \left(1+|\zeta|^{2}\right)-\ln \zeta_{\bar{z}}-\ln \bar{\zeta}_{z}=-\ln \frac{\left|\zeta_{\bar{z}}\right|^{2}}{\left(1+|\zeta|^{2}\right)^{2}}
$$

so that the vorticity equation (22) is satisfied automatically. But we notice that (101) is the general solution of the Liouville equation

$$
\Delta \psi=8 \mathrm{e}^{-\psi} .
$$

If one chooses $\zeta=\prod_{i=1}^{N}\left(\bar{z}-\bar{z}_{i}\right)$ with $N$ simple zeroes in the complex plane then it determines $N$ static vortices located in the plane at zeroes of this function [34]. To have static vortex lattices periodic in $x$ direction we can consider

$$
\zeta=\prod_{i=1}^{N} \sin \left(\bar{z}-\bar{z}_{i}\right)
$$

It determines $N$ time independent magnetic vortex lattices. For $N=1$ the stream function

$$
\psi=\ln \frac{\left(1+|\sin z|^{2}\right)^{2}}{|\cos z|^{2}}
$$

describes periodic in $x$ static lattice of vortices. If we choose $\zeta=\bar{z} \sin \bar{z}$ then the stream function

$$
\psi=\ln \frac{\left(1+|z|^{2}|\sin z|^{2}\right)^{2}}{|\sin z+z \cos z|^{2}}
$$

describes single vortex imposed on vortex lattice.

\section{Conclusions}

To solve the problem of magnetic vortices in a planar spin liquid model, first we found holomorphic reductions of the model and showed that evolution equation at this reduction becomes the linear anti-holomorphic Schrödinger equa- 
tion. Analogy of the anti-holomorphic Cole-Hopf transformation with the well known hydrodynamical relation between the complex velocity and the complex potential, leaded us to formulation of the complex Burgers' equation with integrable $N$ vortex dynamics. We found that vortices correspond to zeroes of the complex Schrödinger equation. This allowed us to construct $N$ vortex configurations, vortex chain lattices and their mutual dynamics in terms of the complex Hermite polynomials. By mapping our vortex problem to $\mathrm{N}$-particle problem, the complexified CalogeroMoser system, we showed its integrability and the Hamiltonian structure.

Finally, we note that the holomorphic Hopf equation

$$
\mathrm{i} u_{t}+u u_{z}=0,
$$

which corresponds to the dispersionless limit of the holomorphic Burgers' equation (24), has been considered very recently as nonlinear bosonization in quantum hydrodynamics for description of quantum shock waves in edge states of fractional quantum Hall effect [40]. The weak solution of this equation for point vortices with strength $\Gamma_{1}, \ldots, \Gamma_{N}$, so that

$$
\operatorname{rot} u=\sum_{k=1}^{N} \Gamma_{k} \delta\left(x-x_{k}(t)\right) \delta\left(y-y_{k}(t)\right)
$$

gives the following vortex system [43]

$$
\frac{\mathrm{d} z_{k}}{\mathrm{~d} t}=4 \mathrm{i} \sum_{l=1,(l \neq k)}^{N} \frac{\Gamma_{l}}{z_{k}-z_{l}}, \quad k=1, \ldots, N .
$$

When all the vortex strengths are equal $\Gamma_{1}=\cdots=\Gamma_{N}$ then this system reduces to (76) and is integrable. However, in the general case the system is not known to be integrable. In particular, for $N=3$ the system with constraint $\Gamma_{1}=\Gamma_{2} \neq \Gamma_{3}$ has been studied recently in [41] to explain the transition from regular to irregular motion as travel on the Riemann surface.

\section{Acknowledgments}

One of the authors (O.P.) thanks F. Calogero and M. Sommacal for useful remarks. This work was partially supported by TUBITAK under the Grant No. 106T447.

\section{References}

[1] Kozlov VV. General vortex theory. Reg. Chaot. Dynamics. Izevsk, 1998.

[2] Helmholtz H. On integrals of the hydrodynamical equations which express vortex motion. Phil Mag 1867;33:485-512.

[3] Kelvin L. On vortex motion. Trans Roy Soc Edin 1869;25:217-60.

[4] Kirchoff GR. Vorlesungen uber mathematische physik mechanik. Teubner: Leipzig; 1876. p. 466.

[5] Gröbli W. Specielle probleme über die bewegung geradliniger paralleler wirbelfäden. Zurich und Furrer; 1877. p. 86.

[6] Lomonaco S. The modern legacies of Thomson's atomic vortex theory in classical electrodynamics. Proc Symp Appl Math 1996;51:145-66.

[7] Ziglin SL. The nonintegrability of the problem on the motion of four vortices of finite strengths. Physica D4 1982;2:268-9.

[8] Lushnikov PM. Exactly integrable dynamics of interface between ideal fluid and light viscous fluid. Phys Lett A 2004;329(1-2):49-54.

[9] Saffman PG. Vortex dynamics. Cambridge: Cambridge University Press; 1992.

[10] Poincaré H. In: Carré G, editor. Théory des tourbillons. Paris: Cours de la Faculté des Sciences de Paris; 1893.

[11] Meleshko VV, Van Heijst GJF. On Chapylgin's investigations of two-dimensional vortex structures in an inviscid fluid. J Fluid Mech 1994;272:157-82.

[12] Borisov AV, Mamaev IS. Mathematical methods of vortex structure dynamics. Moscow: Izesk; 2003 [in Russian].

[13] Kuratsuji H, Yabu H. Force on a vortex in ferromagnet model and the properties of vortex configurations. J Phys A 1998:31.

[14] Komineas S, Papanicolaou N. Vortex dynamics in two-dimensional antiferro-magnets. Nonlinearity 1998;11:265-90.

[15] Ho T Mermin ND. Equilibrium order parameters and chemical potentials in rotating superfluids. Phys Rev E 1980;21:5190-7.

[16] Mermin ND, Ho T. Circulation and angular momentum in the a phase of superfluid Helium-3. Phys Rev Lett 1976;36:594.

[17] Ezawa ZF. Quantum hall effects. Singapore: World Scientific; 2000. p. 94-110.

[18] Thess A, Zikanov O, Nepomnyashchy A. Finite-time singularity in the vortex dynamics of a string. Phys Rev E 1999:59.

[19] Correa DH, Moreno EF, Schaposnik FA, Lazano FA. Particle vortex dynamics in noncommutative space. JHEP 0111034 2001:1-11. 
[20] Pismen LM. Vortices in nonlinear fields. New York: Clarendon Press; 1999. p. 91-278.

[21] El Naschie MS. Holographic dimensional reduction: center manifold theorem and E-infinity. Chaos, Solitons \& Fractals 2006;29:816-22.

[22] El Naschie MS. Radioactive decay and the structure of $\mathscr{E}^{(\infty)}$ quantum spacetime. Chaos, Solitons \& Fractals 1999;10:17-23.

[23] da Cruz W. The Hausdorff dimension of fractal sets and fractional quantum Hall effect. Chaos, Solitons \& Fractals 2003;17:975-9.

[24] El Naschie MS. COBE satellite measurement, Cantorian space and cosmic strings. Chaos, Solitons \& Fractals 1997;8:847-50.

[25] Tchernyshyov O, Chern GW. Fractional vortices and composite domain walls in flat nanomagnets. Phys Rev Lett $2005 ; 95: 197204$.

[26] El Naschie MS. Nanotechnology for the developing world. Chaos, Solitons \& Fractals 2006;30:769-73.

[27] Lavrentiev MA, Shabat BV. Hydrodynamic problems and their mathematical models. Moscow: Nauka; 1973 [in Russian].

[28] Martina L, Pashaev OK, Soliani G. Bilinearization of multidimensional topological magnets. J Phys A 1994:27.

[29] Ishimori Y. Multi-vortex solutions of a two-dimensional nonlinear wave equation. Prog Theoret Phys 1984:72.

[30] Calogero F. Motion of poles and zeros of special solutions of nonlinear and linear partial differential equations and related solvable many-body problems. Il Nuovo Cimento 1978:43B.

[31] Perelomov AM. Integrable systems of classical mechanics and Lie algebras. Basel: Birkhäuser; 1990. p. 1.

[32] Lipovskii VD, Shirokov AV. Example of gauge equivalence of multidimensional integrable equations. Func Anal Appl 1989;23:65.

[33] Makhankov VG, Pashaev OK. Integrable pseudospin models in condensed matter. Sov Sci Rev Math Phys 1992;9:131-9.

[34] Martina L, Pashaev OK, Soliani G. Static vortex solutions and singular auxiliary field in the Ishimori model. Inverse Problems 1994;10:7-10.

[35] Martina L, Pashaev OK, Soliani G. On topological soliton dynamics in multidimensional ferromagnetic continuum. Theoret Math Phys 1994;99:726.

[36] Konopelchenko BG. Nonlinear integrable equations. Berlin: Springer; 1987.

[37] Pashaev OK. Integrable Chern-Simons gauge field theory in 2+ 1 dimensions. Mod Phys Lett A 1996;11:1713-28.

[38] Arfken GB, Weber HJ. Mathematical methods for physicists. New York: Academic Press; 1995.

[39] Choodnovsky DV, Choodnovsky GV. Pole expansions of nonlinear partial differential equations. Il Nuovo Cimento 1977:40B.

[40] Abanov AB, Wiegmann PB. Quantum hydrodynamics, the quantum Benjamin-Ono equation, and the Calogero model. Phys Rev Lett 2005:95.

[41] Calogero F, Gomez-Ullate D, Santini PM, Sommacal M. The transition from regular to irregular motions, explained as travel on Riemann surfaces. J Phys A 2005;38:8873-96.

[42] Calogero F. Classical many-body problems amenable to exact treatments. Berlin: Springer; 2001.

[43] Pashaev OK, Gurkan ZN. Hydrodynamical vortices in holomorphic Burgers'-Hopf equation, in preparation. 